\title{
Surface Nanoscale Axial Photonics (SNAP) at the Silica Microcapillary with Ultrathin Wall
}

\author{
T. Hamidfar ${ }^{(1,2)}$, A. Dmitriev ${ }^{(1)}$, B. Magdan ${ }^{(3)}$, P. Bianucci ${ }^{(1)}$, and M. Sumetsky ${ }^{(2)(*)}$ \\ ${ }^{(1)}$ Concordia University, Montreal, Québec H4B 1R6, Canada \\ (2) Aston Institute of Photonic Technologies, Aston University, Birmingham B4 7ET, UK \\ (3) OFS Laboratories, 19 Schoolhouse Road, Somerset, NJ 08873, USA \\ ${ }^{(*)}$ m.sumetsky@aston.ac.uk
}

\begin{abstract}
We demonstrate SNAP microresonators fabricated in silica capillary fiber with ultrathin walls by local annealing with a focused $\mathrm{CO}_{2}$ laser and internal etching with hydrofluoric acid. We investigate the introduced capillary wall nonuniformity and demonstrate the feasibility of advanced microfluidic sensing with SNAP microresonators.
\end{abstract}

Keywords—microfluidics, microresonator, optical sensing.

\section{INTRODUCTION}

The Surface Nanoscale Axial Photonics (SNAP) platform [1] enables fabrication of resonant ultralow loss photonics structures at the surface of an optical fiber with unprecedented precision currently approaching 0.1 angstrom [2]. The idea of SNAP consists in exploring whispering gallery modes (WGMs) which slowly propagate along the surface of an optical fiber. The wavelength of WGMs considered in SNAP is very close to their cutoff wavelength. For this reason, these modes are slow, i.e., their axial speed and propagation constant are small. As the result, the distribution of WGMs along the fiber axis can be controlled by extremely small nanoscale variation of the fiber radius. Potential applications of the SNAP platform include slow light delay lines, buffers and signal processors [3, 4], frequency comb generators [5], optomechanical devices [6], and microfluidic sensors and manipulators [7].

This paper presents the first experimental demonstration of SNAP microresonators at a thin optical capillary fiber and establishes the groundwork for the development of SNAP microfluidic sensors [7]. Microfluidic sensing with optical WGMs circulating along the uniform capillary has been proposed and demonstrated in Ref. [8] and developed in numerous publications $[9,10]$. Employing a SNAP resonator at the capillary surface allows to significantly advance this method. In particular, the approach suggested in [7] potentially enables the reconstruction of the spatial distribution of refractive index of fluid flowing inside the capillary from the resonator spectrum and simultaneous sensing and manipulation of fluid components.

\section{EXPERIMENT}

The device fabricated and investigated in this paper is illustrated in Fig. 1. It consists of a silica fiber capillary with external radius $r_{0}=21 \mu \mathrm{m}$ and initial wall thickness of $6 \mu \mathrm{m}$. WGMs are excited in the capillary with a transverse biconical fiber taper, which has a micron-diameter waist (microfiber) positioned in direct contact to the capillary. The taper was fabricated from a conventional single mode fiber and connected to the laser source and optical spectrum analyzer (OSA) to measure the resonant spectrum of WGMs.
Following [7] we introduced an asymmetric SNAP microresonator at the capillary surface by local annealing with a focused $\mathrm{CO}_{2}$ laser beam. The WGM spectra of this resonator were measured at microfiber positions spaced by $20 \mu \mathrm{m}$ along the capillary axis (Fig. 2(a)). The length of the resonator was $\sim$ $300 \mu \mathrm{m}$ and the height of the introduced effective radius variation was calculated as $\Delta r_{0} \sim \Delta \lambda_{0} r_{0} / \lambda_{0} \sim 2 \mathrm{~nm}$. Here $\Delta \lambda_{0} \sim 0.15 \mathrm{~nm}$ is the spectral height of the resonator estimated from Fig. 2(a) and $\lambda_{0} \sim 1.57 \mu \mathrm{m}$ is the wavelength considered. The spectral surface plot shown in Fig. 2(a) corresponds to the vicinity of a cutoff wavelength having azimuthal quantum number $m \sim 2 \pi n_{c} r_{0} / \lambda_{0}$ and fundamental radial quantum number $p=0$. For a silica capillary with refractive index $n_{c} \sim 1.5$, we have $m \sim 100$. Similar spectral distributions are observed in the vicinity of cutoff wavelengths corresponding to other quantum numbers $m$ and $p$.

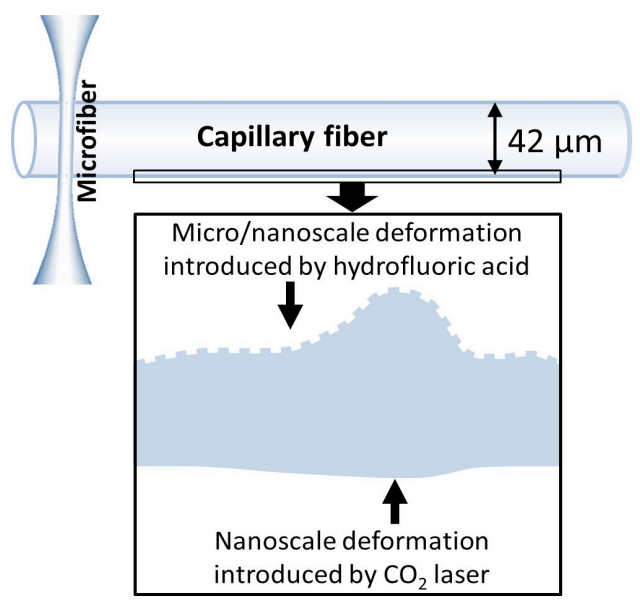

Fig. 1. Illustration of a capillary fiber coupled to a microfiber taper processed with a $\mathrm{CO}_{2}$ laser and hydrofluoric acid. Inset: magnified cross-section of the capillary wall (not to scale).

For the capillary with the original wall thickness $\Delta r_{w}=6$ $\mu \mathrm{m}$, the WGM spectrum of the SNAP microresonator was not sensitive to the presence of water used as microfluid in our experiment. In order to enhance the sensitivity, we etched the internal capillary surface with the hydrofluoric acid and simultaneously controlled the resonance spectrum [8]. For the results of experiment described below, the etching process was stopped after the shift of axial resonances with quantum numbers $q=0,1,2, \ldots$ adjacent to the cutoff wavelength with fundamental radial quantum number $p=0$ (Fig. 2(a)) was observed. 

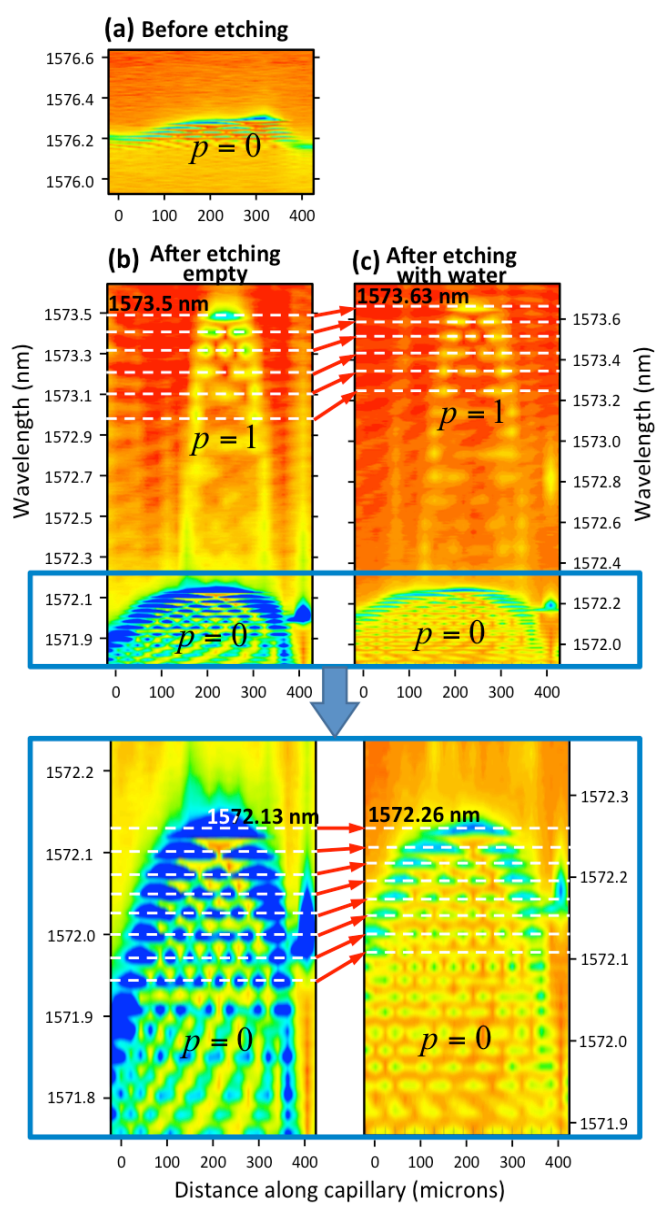

Fig. 2. Surface spectral plots experimentally measured with $20 \mu \mathrm{m}$ resolution along the capillary axis. (a) - SNAP resonator before etching for the $p=0$ WGMs (the $p=1$ WGMs not shown here have similar spectral distribution). SNAP resonator after etching for the $p=0$ (bottom) and $p=1$ (top) WGMs: (b) - empty capillary (c) capillary filled with water. Inset: magnified plots of $p=0$ spectra.

Comparison of the SNAP resonator spectra before etching (Fig.2(a)) and after etching (empty capillary in Fig. 2(b) and capillary filled with water in Fig. 2(c)) shows significant modifications. Intriguingly, while the axial WGMs resonances adjacent to the cutoff wavelength with the fundamental radial number $p=0$ (bottom) experience relatively small distortion, the axial resonances of WGMs with a larger radial quantum number assumed as $p=1$ (top) change dramatically. It is seen that the $p=0$ axial resonances are narrower, i.e., much less lossy than the $p=1$ resonances. This is explained by the fact that the width of the $p=1$ WGMs along the radial direction is greater. Therefore, these modes have stronger overlap with the internal surface of the capillary, which is rougher than the external surface due to the etching process. While the fundamental axial mode with $q=0$ and $p=1$ did not experience strong changes, the dramatic shrinking of the $p=1$ WGMs with larger axial quantum numbers $q$ along the axial direction can be explained by the nonuniformity introduced by hydrofluoric etching. We suspect that, in addition to the nanoscale variation of the capillary wall thickness, the local $\mathrm{CO}_{2}$ laser annealing deforms the wall as a whole. This deformation disturbs the hydrofluoric acid flow in the process of etching and leads to the creation of a bump (local thickening) of the capillary wall illustrated in the inset of
Fig.1. While the $p=0$ WGMs have small overlap with the internal wall surface and, therefore, are not noticeably sensitive to this deformation, the bump causes the additional localization of the $p=1$ modes evident from Fig. 2(b) and (c). Here we were not able to determine the full height of the internally created bump since the spectrum of the $p=1$ WGMs became blurry and unclear for large axial quantum numbers $q$.

Comparison of Fig. 2(b) and (c) demonstrates the effect of the presence of water on the spectra of resonances with axial quantum numbers $q=0,1,2, \ldots$ and radial quantum numbers $p=0$ and (presumably) $p=1$. Notice that, after adding water, the spectrum of the SNAP microresonator was shifted as a whole by $0.13 \mathrm{~nm}$. We hypothesize that this shift happened due to mechanical deformation of the capillary pressurized by water. Remarkably, the separation of the resonant wavelength of the fundamental axial modes with $q=0$ (the top axial resonances of $p=0$ and $p=1$ series) did not change. This fact confirms, again, that the capillary wall thickness in the region of localization of fundamental axial WGMs was large enough. However, the effect of water on the resonance shift grew with axial quantum number $q$ (i.e., with the WGM axial width). As expected, this shift (indicated by tilted red arrows connecting the resonances in Fig. 2(b) and (c)) is significantly greater for the $p=1$ series.

\section{SUMMARY}

We have experimentally demonstrated a SNAP microresonator created at the surface of a capillary fiber by local annealing with a focused $\mathrm{CO}_{2}$ laser beam. In order to ensure the microfluidic sensing with this resonator, we have thinned the capillary wall internally by etching with hydrofluoric acid. As the result, we observed the variation of SNAP microresonator spectra in response to the presence of water inside the capillary. The observed shifts of resonances strongly depended on the spatial distribution of corresponding WGMs. In addition, we detected the nonuniformity of the internal capillary surface introduced by etching. Overall, this demonstration paves the groundwork for the advanced microfluidic sensing with SNAP microresonators.

\section{REFERENCES}

[1] M. Sumetsky, "Nanophotonics of optical fibers," Nanophotonics 2, 393 (2013).

[2] N. A. Toropov and M. Sumetsky, "Permanent matching of coupled optical bottle resonators with better than $0.16 \mathrm{GHz}$ precision," Opt. Lett. 41, 2278 (2016).

[3] M. Sumetsky, "Delay of light in an optical bottle resonator with nanoscale radius variation: dispersionless, broadband, and low loss," Phys. Rev. Lett. 111, 163901 (2013).

[4] M. Sumetsky, "Microscopic optical buffering in a harmonic potential," Sci. Rep. 5, 18569 (2015).

[5] V. Dvoyrin and M. Sumetsky, "Bottle microresonator broadband and lowrepetition-rate frequency comb generator,” Opt. Lett. 41, 5547 (2016).

[6] M. Sumetsky, "Optical bottle versus acoustic bottle and antibottle resonators," Opt. Lett. 42, 923 (2017).

[7] M. Sumetsky, "Slow light optofluidics: a proposal," Opt. Lett. 39, 5578 (2014).

[8] I. M. White, H. Oveys, and X. Fan, "Liquid-core optical ring-resonator sensors," Opt. Lett. 31, 1319 (2006).

[9] X. Fan and I. M. White, "Optofluidic microsystems for chemical and biological analysis," Nat. Photonics 5, 591 (2011).

[10] T. Reynolds, N. Riesen, A. Meldrum, X. Fan, J. M. M. Hall, T. M. Monro, and A. Francois, "Fluorescent and lasing whispering gallery mode microresonators for sensing applications,' Laser Photonics Rev. 11, 1600265 (2017). 